\title{
On the Link between Electron Shadowing and Charging Damage
}

\author{
Gyeong S. Hwang and Konstantinos P. Giapis* \\ Division of Chemistry and Chemical Engineering, California Institute of Technology, Pasadena, CA 91125
}

(January 13, 1997)

\begin{abstract}
Charging and topography evolution simulations during plasma etching of high aspect ratio lineand-space patterns reveal that electron shadowing of the sidewalls critically affects charging damage. Decreasing the degree of electron shadowing by using thinner masks decreases the potentials of the etched features with a concomitant reduction in catastrophic tunneling currents through underlying thin gate oxides. Simultaneously, the potential distribution in the trench changes, significantly perturbing the local ion dynamics which, in turn, cause the notching effect to worsen. Since the latter can be reduced independently by selecting an appropriate etch chemistry, the use of thinner (hard) masks is predicted to be advantageous for the prevention of gate oxide failure.
\end{abstract}

Pattern-dependent charging is rapidly becoming one of the most serious issues to confront plasma processing of advanced ultra-large scale integrated (ULSI) circuits. When etching gate electrodes in high-density plasmas, for example, it has been shown that charging of the electrodes can lead to large tunneling currents through the underlying thin gate oxide causing degradation [1]. The charge buildup is a result of large fluxes of deflected ions towards the sidewalls, occurring when insulating trench bottoms acquire large positive potentials [2]. Such a perturbation in the local ion dynamics can also cause lateral etching and significant sidewall profile distortion (notching) [2-4]. Catastrophic tunneling currents and notching constitute charge-induced damage, which is expected to worsen as the incessant quest for miniaturization of integrated circuits causes the aspect ratio of devices to increase [5]. Given the severity of the problem, there is a trend to replace photoresist masks with hard (oxide) masks which can be made thinner, thus keeping aspectratio-dependent problems under control despite the decrease in device dimensions. For a constant aspect ratio, however, the mask replacement effectively changes the degree of electron shadowing of the sidewalls, with important consequences for charging and notching.

When plasma etching line-and-space (L\&S) patterns of polysilicon-on-insulator structures, the feature aspect ratio (etch depth over line spacing) influences critically the ability of the electrons to reach the trench bottom. This is partly a result of geometric shadowing of the more isotropic electrons and partly due to the local electrostatics in the trench, which adjust the bottom and sidewall potentials so that electrons can be attracted to balance the ion current to the various surfaces [5]. When the aspect ratio is constant but the mask thickness is varied, the entrance potentials do not change and the electron flux into the trench is not affected. However, the concomitant change in the polysilicon thickness alters the percentage of the sidewalls that is electrically conductive. It becomes easier for larger polysilicon sidewalls to receive electrons and, thus, to acquire a lower potential. A reduction in the potential difference between the gate electrode and the substrate-separated by a thin oxide layer, decreases the probability for oxide breakdown. Therefore, use of a thinner mask bears promise for a reduction in charging damage during etching of metaloxide-semiconductor structures, even when the aspect ratio is high $(\geq 4)$. This conjecture is a strong motive for a study of the influence of the mask thickness on charging and notching, which has yet to be performed experimentally or modeled theoretically. The parameter of interest is the ratio of the mask thickness to the trench width, defined here as the "shadowing" ratio (SR). Although a part of the feature aspect ratio, the shadowing ratio can be varied independently from the latter. To isolate the effect of the shadowing ratio on charging damage, the aspect ratio is kept constant at a high value of $4: 1$ throughout the present study.

The plasma conditions determine the extent of charging damage in a particular mìcrostructure [3]. Here, we employ a low-pressure chlorine plasma with a density of $1 \times 10^{12} \mathrm{~cm}^{-3}$. The sheath voltage is $37+30 \sin w t$ (Volts), where $\omega=400 \mathrm{kHz}$ is the $\mathrm{rf}$ bias frequency. The ion and electron temperatures are taken to be 0.5 and $4.0 \mathrm{eV}$, respectively. At the high plasma density considered, the chlorine is assumed to be $100 \%$ dissociated. The dense structure to be simulated (Fig. 1) borders a widely open area and consists of $0.3 \mu \mathrm{m}$ lines separated by $0.3 \mu \mathrm{m}$ spaces (trenches); the lines are not electrically connected. At the onset of overetching, each feature consists of insulating photoresist onto poly-Si; the features are formed on top of a uniform layer of $\mathrm{SiO}_{2}$, thick enough $(>100$ $\mathrm{nm}$ ) to prevent tunneling currents to the Si substrate. Two cases with different photoresist and poly-Si thicknesses will be considered at a constant aspect ratio of $4: 1$. When the features consist of $0.9 \mu \mathrm{m}$ photoresist onto 0.3 $\mu \mathrm{m}$ poly-Si (Fig. 1a), the structure is representative of $0.3 \mu \mathrm{m}$ device rule. A structure with features of $0.3 \mu \mathrm{m}$ photoresist (or hard mask) onto $0.9 \mu \mathrm{m}$ poly-Si (Fig. 1b) is not conventional for $0.3 \mu \mathrm{m}$ device rule. However, if aspect ratio scaling holds, the latter structure could be representative of $0.1 \mu \mathrm{m}$ device rule, by forming $0.1 \mu \mathrm{m}$ hard mask $\left(\mathrm{SiO}_{2}\right)$ onto $0.3 \mu \mathrm{m}$ poly-Si. The poly-Si is 

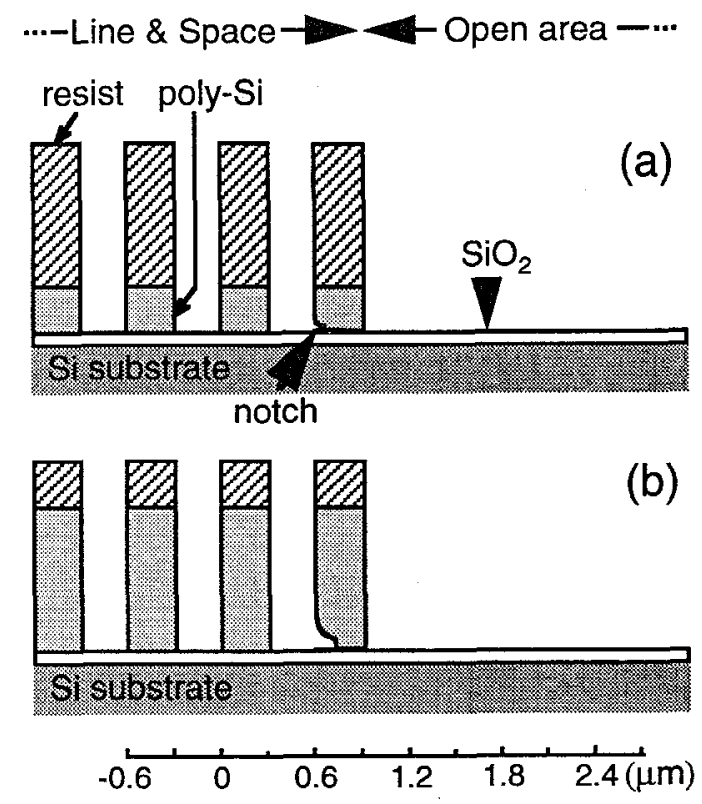

FIG. 1. Schematic depiction of the line-and-space structures considered in the simulation for a shadowing ratio of (a) 3:1 and (b) 1:1. The open areas are assumed to be infinite.

assumed to be heavily doped (electrically conductive).

Understanding charging damage requires detailed modeling and simulation of ion and electron dynamics in plasmas, through sheaths, and as they impinge at various microstructure surfaces where they cause charging, physical sputtering and chemical reactions, or undergo inelastic scattering. A predictive feature-scale simulation that discusses these steps and simplified models to describe them has been presented elsewhere [2]. In this report, we extend our numerical simulation to investigate the effect of a thinner mask on microstructure charging and sidewall profile distortion. Briefly, charging is decoupled from etching for the short time required for the former to reach steady-state. Realistic ion and electron energy and angular distributions at the wafer are calculated from sheath theory. Then, charged particles are followed as they impinge at various surfaces, where they transfer their charge. Charge deposition creates local electric fields which, in turn, alter ion trajectories. The Laplace equation is solved repeatedly in the microstructure spaces to account for the evolution of the electric fields as more charge accumulates. Steady-state is established when the potential distribution along the bottom $\mathrm{SiO}_{2}$ surface no longer changes. For the present calculation, surface currents and secondary electron emission are both neglected. As mentioned earlier, tunneling currents through the thick gate oxide are also disregarded; thus, the present study focuses on a worst-case scenario, aiming to reveal where breakdown is most likely to occur from excessive charging.
The charged particle dynamics in the trench next to the edge line are essential in understanding what happens to the sidewall potentials. The steady-state potential distribution in the trench, plotted in Fig. 2 for various mask thicknesses at constant aspect ratio, can help reveal the perturbation in the local ion dynamics that follows the change in the mask thickness. These plots illustrate the potential felt by the ions as they traverse the trench region confined by the poly-Si sidewalls. We have previously shown that an asymmetric potential distribution at the trench bottom, with a pronounced peak near the inner sidewall foot of the outermost line, is critical for the formation of the notch [2]. Indeed, when electron shadowing of the sidewalls is significant $(\mathrm{SR}=3: 1)$, the potential distribution in the outermost trench, shown in Fig. 2a, has the "classic" form that forecasts notching at the inner side of the outermost poly-Si line. Remarkably, when the mask thickness is decreased ( $S R=1: 1)$, the potential distribution in the outermost trench, shown in Fig. 2b, becomes bimodal. The peak nearer to the edge line shifts towards the trench middle and broadens considerably, while a new peak appears by the sidewall foot of the neighboring line. These observations forecast the formation of a larger notch at the inner side of the edge line when the mask gets thinner. They also suggest that notching could also occur at the neighboring line. But how is it possible to have two potential distribution maxima? Key to the formation of a potential maximum at the trench bottom is a low poly-Si potential. When electron shadowing is significant (high SR), only the outermost lines can maintain a low potential because they receive electrons from the side facing the open area [5]. All other lines in an isolated L\&S structure acquire a much larger potential since few electrons can be supplied through the trench entrance. Indeed, for $\mathrm{SR}=3: 1$, the calculated potentials of the edge line and its neighboring line are 6.8 and $31.8 \mathrm{~V}$, respectively. A potential maximum forms near the inner sidewall foot of the outermost line as a result of the deflection of ion trajectories towards the lower potential of that line. When thinner masks are used (low SR), electron shadowing decreases; therefore, more electrons can be supplied to the intermediate poly-Si lines through the trench openings, decreasing their potentials. For $\mathrm{SR}=1: 1$, the corresponding potentials are calculated to be 2.7 and $10.6 \mathrm{~V}$, respectively. The relatively low value $(10.6 \mathrm{~V})$ of the potential of the neighboring line is responsible for the appearance of the second peak in the distribution of Fig. $2 \mathrm{~b}$. Note that if the potentials of the two lines become equal and remain low, symmetric peaks will appear, as in the case of electrically connected lines [2]. The dramatic reduction in the line potentials that follows the decrease in the shadowing ratio suggests that thinner gate oxides can be used without fear for catastrophic leakage currents.

The steady-state charging potentials determine the ion dynamics-in the microstructure. Reactions at the poly- 

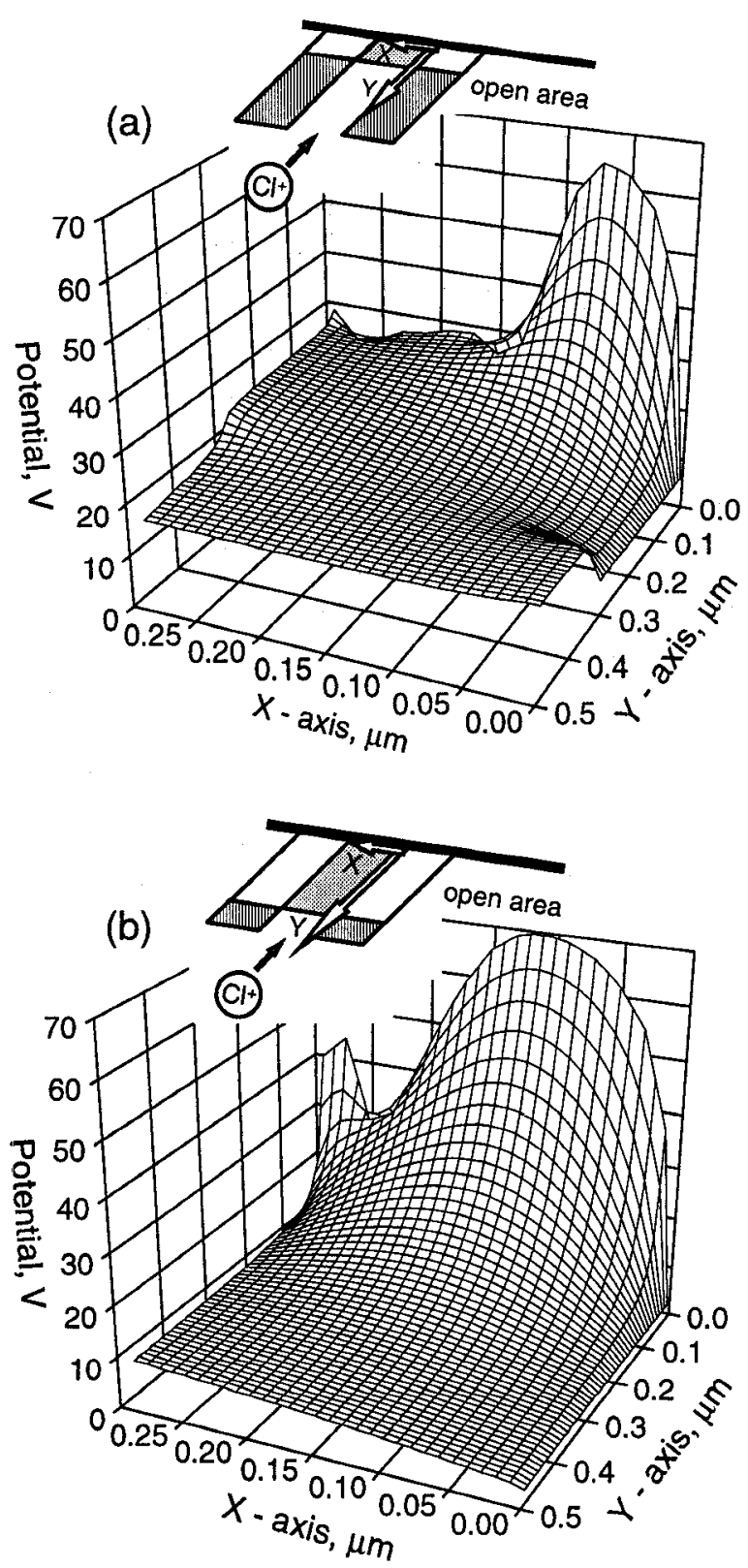

FIG. 2. Two-dimensional charging potential distributions in the space of the outermost trench for a shadowing ratio of (a) 3:1 and (b) 1:1. The inset illustrates the area of interest (extencling $0.5 \mu \mathrm{m}$ from the trench bottom) and defines the origin for the potential surface. The microstructure has been rotated to allow for a more convenient description of ion motion in the trench. The arrows show the direction of ions as they approach the potential surface. In (a), notice the potential perturbation at the region near the photoresist/poly-Si interface $(y=0.3 \mu \mathrm{m})$.

Si sidewalls and scattering at the $\mathrm{SiO}_{2}$ surface determine how the profile will evolve. The profile evolution simulation combines the ion dynamics with reactions and scattering and is performed as described elsewhere [2]. As etching of the sidewall proceeds, charging of the exposed $\mathrm{SiO}_{2}$ leads to significant forward deflection of ener- getic ions which contribute to notching. Thus, the etching simulation becomes coupled to transient charging in the etched area, which complicates the calculation. The problem becomes tractable by solving for a new potential distribution every time the notch apex is advanced to the next cell layer. Sputtering and chemical etching of $\mathrm{SiO}_{2}$ are presumed to be insignificant. Etching of the poly-Si is assumed to be ion-limited while spontaneous etching by $\mathrm{Cl}$ atoms is neglected. Material is removed from the surface by physical sputtering of chlorinated $\mathrm{SiCl}_{x}$ moieties; the energy and angular dependence of the etch yield has been discussed before [2]. Inelastic scattering on poly$\mathrm{Si}$ is neglected as most of the scattered atoms impinge subsequently on $\mathrm{SiO}_{2}$. However, direct inelastic scattering on the $\mathrm{Cl}$-covered $\mathrm{SiO}_{2}$ surface must be included as all of the scattered atoms impinge subsequently on poly$\mathrm{Si}$. Only specular reflection is considered with an energy transfer that follows hard-sphere collision kinematics [6] with a gas-atom to surface mass ratio of 1.0.

Profile evolution simulations have been performed for the two cases and the profiles obtained for a fixed number of ions [7] entering the outermost trench are shown in Fig. 3. Remarkably, the notch depth at the edge line increases from 95 to $160 \mathrm{~nm}$ when decreasing the mask thickness from 0.9 to $0.3 \mu \mathrm{m}$. A minuscule notch also appears at the sidewall facing the edge line (not shown). These observations can be fully justified in view of the corresponding trench potential distribution (see Fig. 2). Calculation of the normalized flux [8] and average energy for ions impinging at the bottom surface, and the poly$\mathrm{Si}$ sidewalls confining the outermost trench [9], listed in Table I, provides further insight into how the charging potentials influence the local ion dynamics to increase notching. The larger and broader potential maximum by the edge line of the thin mask case (Fig. 2b) causes defle-

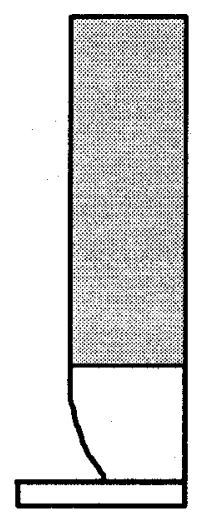

(a)

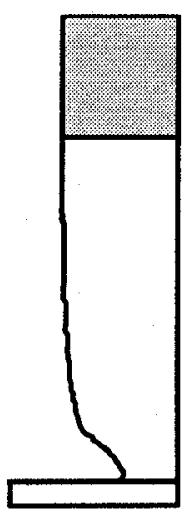

(b)
FIG. 3. Notch profile at the inner sidewall of the outermost line for a shadowing ratio of (a) 3:1 and (b) 1:1, predicted for the same (arbitrary) overetching time. Etching of the outer sidewall is not simulated. The aspect ratio has been preserved. 
Table 1 . The normalized flux and average energy of ions bombarding the indicated surfaces. The data for the sidewalls are restricted to the bottom $0.18 \mu \mathrm{m}$ (notch area).

\begin{tabular}{c||c|c|c||c|c}
\hline \multicolumn{1}{c||}{} & \multicolumn{2}{c||}{ Ion Flux (arb. u.) } & \multicolumn{2}{c}{ Avg. Energy (eV) } \\
\hline $\begin{array}{c}\text { Shadowing } \\
\text { ratio }\end{array}$ & $\begin{array}{c}\text { Trench } \\
\text { bottom }\end{array}$ & $\begin{array}{c}\text { Neighbor. } \\
\text { line }\end{array}$ & $\begin{array}{c}\text { Edge } \\
\text { line }\end{array}$ & $\begin{array}{c}\text { Neighbor. } \\
\text { line }\end{array}$ & $\begin{array}{l}\text { Edge } \\
\text { line }\end{array}$ \\
\hline \hline $3: 1$ & 0.200 & 0.006 & 0.176 & 28.6 & 32.1 \\
$1: 1$ & 0.070 & 0.036 & 0.215 & 30.9 & 40.9 \\
\hline
\end{tabular}

ction of more ions towards the sidewalls at the cost of the ion flux to the bottom surface $\left(\mathrm{SiO}_{2}\right)$. In addition, the average translational energy to the inner sidewall of the edge line increases considerably when decreasing the shadowing ratio from 3:1 to $1: 1$. Thus, notching is predicted to become more severe as the mask thickness decreases. The wider notch is a consequence of the broader and larger potential maximum, which deflects ions farther from the trench bottom so that they impinge higher up along the sidewall. Smaller potential maxima result typically in narrower and sharper notches [10]. Although the relative increase in the ion flux to the neighboring poly-Si sidewall is substantial (Table I), the absolute value of the flux is very small to cause significant notching at that location.

In conclusion, charging and etching simulations in high aspect ratio. L\&S structures reveal that electron shadowing critically influences charging damage during overetching of polysilicon-on-insulator structures in uniform high-density plasmas. For a fixed aspect ratio, decreasing mask thickness reduces electron shadowing of the poly-Si sidewalls, which can now be maintained at a lower charging potential by electrons supplied through the trench entrance. A smaller electric field develops across the underlying gate oxide, thus decreasing the probability for catastrophic tunneling currents. Simultaneously, the lower charging potentials of the poly-Si sidewalls cause the potential distribution at the trench bottom to broaden and shift towards the trench middle. The flux and energy of ions to the inner sidewall of the outermost line increases, thus worsening notching at that location. However, since notching can be minimized by changing the overetch chemistry [2], the use of thinner (hard) masks-as device dimensions shrink-should be beneficial for the reduction of charge-induced oxide degradation.
* E-mail: giapis@cheme.caltech.edu

[1] K. Hashimoto, Jpn. J. Appl. Phys. 32, 6109 (1993); ibid. 33, 6013 (1994).

[2] G. S. Hwang and K. P. Giapis, J. Vac. Sci. Technol. B 15, in print (Jan/Feb 1997).

[3] N. Fujiwara, T. Maruyama, M. Yoneda, Jpn. J. Appl. Phys. 34, 2095 (1995); ibid. 35, 2450 (1996).

[4] T. Nozawa, T. Kinoshita, T. Nishizuka, A. Narai, T. Inoue, and A. Nakaue, Jpn. J. Appl. Phys. 34, 2107 (1995).

[5] G. S. Hwang and K. P. Giapis, J. Appl. Phys. (submitted).

[6] G. S. Hwang, C. M. Anderson, M. J. Gordon, T. A: Moore, T. K. Minton, K. P. Giapis, Phys. Rev. Lett. 77, 3049 (1996).

[7] No overetching time is stated because it is impossible to calibrate the reactive ion flux without an etching experiment of a structure similar to the simulated one.

[8] The flux has been normalized with respect to the total ion flux entering the outermost trench.

[9] Since the poly-Si sidewall area effectively increases when the mask gets thinner at constant aspect ratio, the flux and average energy data in Table I are restricted to the first $0.18 \mu \mathrm{m}$ from the poly-Si/ $\mathrm{SiO}_{2}$ interface-the "notching" area-to make the comparison meaningful.

[10] G. S. Hwang and K. P. Giapis, Appl. Phys. Lett. (submitted). 